\title{
Electron Transfer Properties from Atomistic Simulations and Density Functional Theory
}

\author{
Joost VandeVondele $\S^{\S a}$, Marialore Sulpizi ${ }^{\mathrm{b}}$, and Michiel Sprik ${ }^{\mathrm{b}}$ \\ $\S$ METTLER TOLEDO Award Winner (Oral Presentation)
}

\begin{abstract}
Marcus theory of electron transfer is the quintessential example of a successful theory in physical chemistry. In this paper, we describe the theoretical approach we have adopted to compute key parameters in Marcus theory. In our method, based on molecular dynamics simulations and density functional theory, the redox center and its environment are treated at the same level of theory. Such a detailed atomistic model describes specific solvent-solute interactions, such as hydrogen bonding, explicitly. The quantum chemical nature of our computations enables us to study the effect of chemical modifications of the redox centers and deals accurately with the electronic polarization of the environment. Based on results of previous work, we will illustrate that quantitative agreement with experiment can be obtained for differences in redox potentials and solvent reorganization energies for systems ranging from small organic compounds to proteins in solution.
\end{abstract}

Keywords: Density functional theory · Electron transfer - Marcus theory · Molecular dynamics

\section{Introduction}

Electron transfer (ET) reactions play a crucial role in a number of processes of biological and technological importance. Wellknown examples include cell respiration, photosynthesis, fuel cell catalysis and photovoltaics. ${ }^{[1,2]}$ The efficiency of these processes can be optimized by tuning the ET properties of electron-donor and -acceptor or the pathway between them. The relative stability of the electron at these sites (i.e. differences in redox potentials), and the rate of electron transfer between them are of particular interest, as they reflect directly what is thermodynamically and kinetically feasible. In this paper, we will summarize some of our previous work $^{[3-15]}$ aimed at computing these quantities directly from

\footnotetext{
${ }^{\star}$ Correspondence: Dr. J. VandeVondele

Tel.: + 41446354421

Fax: + 41446356838

E-Mail: vondele@pci.unizh.ch

anstitute of Physical Chemistry

University of Zurich

Winterthurerstrasse 190

$\mathrm{CH}-8057$ Zürich

bDepartment of Chemistry

University of Cambridge

Lensfield Road

CB2 1EW, Cambridge, UK
}

atomistic models using density functional Theory (DFT). The quantum chemical nature of DFT allows the effects of chemical modifications of the redox centers to be studied without a need for prior knowledge or empirical parameterization. This can thus make our method truly predictive.

A key feature of ET reactions, or redox reactions in general, is the crucial role played by the environment. Indeed, oxidation (reduction) potentials are the condensed phase equivalents of ionization potentials (electron affinities) in the gas phase, and these quantities can differ significantly. Our atomistic models explicitly include the environment (e.g. solvent and/or protein) so that not only dielectric properties but also specific interactions, such as hydrogen bonding or conformational changes are taken explicitly into account. Furthermore, the environment is far from being a static spectator. Its fluctuations bring donor and acceptor sites in an energy resonant state, triggering ET, and its ability to relax after ET influences significantly the energetics. In order to probe these fluctuations and relaxations in our computational setup, molecular dynamics is employed to generate a sufficiently large number of representative configurations of solute and solvent.

A major step in the understanding of ET reactions was the formulation by Marcus ${ }^{[16,17]}$ of the rate of electron transfer $\left(\mathrm{k}_{\mathrm{ET}}\right)$ as a simple function of the reaction free energy $(\Delta G)$, the solvent reorganization energy $(\lambda)$ and a proportionality constant $(\kappa)$ depending on the quantum coupling between donor and acceptor states.

$$
k_{E T}=\kappa \exp \left(\frac{-(\lambda+\Delta G)^{2}}{4 \lambda k T}\right)
$$

The fruitful concept that underlies this formula is the assumed harmonic nature of the free energy surface with respect to the reaction coordinate of electron transfer. This restricts the validity of this formula to the range of systems that fall in the linear response regime, which might thus exclude systems that undergo significant changes in conformation or solvation upon electron transfer. Ultimately, the success of this theory is based on its capability to predict and explain experimental results. As will be illustrated in Section 2, our and other groups ${ }^{[18-22]}$ have adopted the central concept of this theory to simplify and guide calculations.

So far we have focused on computing two of the three central parameters in Marcus theory: $\Delta \mathrm{G}$, the driving force of the ET reaction, and $\lambda$, the solvent reorganization energy. Anticipating our results in Section 3 , we find good agreement with experiment for systems ranging from small organic 
compounds to proteins in solution. Whereas our DFT calculations typically involve a few hundred atoms, the latter system was modeled using a DFT description for over 2800 atoms. ${ }^{[15]}$

\section{Atomistic Theory of Electron Transfer}

A central element of our approach to ET so far, is the observation that $\Delta \mathrm{G}$ and $\lambda$ can be obtained from standard electronic ground state calculations, thus avoiding the complexity of excited state calculations, if one focuses on electrochemical half reactions. In this case, a single redox active center is explicitly present in the simulation cell, either in reduced or oxidized form. Such a setup only makes sense if there is no strong coupling between the donor and acceptor site, and is thus most easily applied in the case of long-range electron transfer. The free energy difference between the reduced and oxidized redox state, which we will denote by $\Delta \mathrm{A}$, to indicate that our simulations are at constant volume, can be computed in a number of ways. In the present work, we derive expressions for $\Delta \mathrm{A}$ based on thermodynamic integration, i.e. integrating the reversible work needed to change the system's Hamiltonian linearly from the reduced $\left[\mathrm{H}_{\mathrm{re}}\left(\left\{\mathrm{R}_{\mathrm{i}}\right\}\right)\right]$ to the oxidized $\left[\mathrm{H}_{\mathrm{ox}}\left(\left\{\mathrm{R}_{\mathrm{i}}\right\}\right)\right]$ one. We write

$$
\begin{aligned}
& \Delta A=\int_{0}^{1}\left\langle\frac{\partial H_{\alpha}\left(\left\{R_{i}\right\}\right)}{\partial \alpha}\right\rangle_{\alpha} d \alpha=\int_{0}^{1}\left\langle\Delta E\left(\left\{R_{i}\right\}\right)\right\rangle_{\alpha} d \alpha \\
& H_{\alpha}\left(\left\{R_{i}\right\}\right)=\alpha H_{o x}\left(\left\{R_{i}\right\}\right)+(1-\alpha) H_{r e}\left(\left\{R_{i}\right\}\right) \\
& \frac{\partial H_{\alpha}\left(\left\{R_{i}\right\}\right)}{\partial \alpha}=\Delta E\left(\left\{R_{i}\right\}\right)=H_{o x}\left(\left\{R_{i}\right\}\right)-H_{r e}\left(\left\{R_{i}\right\}\right)
\end{aligned}
$$

where $\mathrm{H}_{\alpha}$ is a Hamiltonian, formed as a linear combination of the two physical Hamiltonians $\mathrm{H}_{\mathrm{ox}}$ and $\mathrm{H}_{\mathrm{re}}$. Its derivate with respect to $\alpha$ is the vertical energy gap $\Delta \mathrm{E}$, a central quantity in the following. The integrand $<\Delta E\left(\left\{R_{i}\right\}\right)>_{\alpha}$ is the canonical average of the vertical energy as obtained from a sampling based on the Hamiltonian $\mathrm{H}_{\alpha}$, for which we will introduce the short hand notation $\Delta \mathrm{E}_{\alpha}$. The above expression for $\Delta \mathrm{A}$ is exact, and practical for actual $a b$ initio

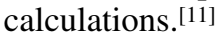

Nevertheless, let us assume that the system is in the Marcus regime, or equivalently in the linear response regime. In this case, the integrand $\Delta \mathrm{E}_{\alpha}$ varies linearly between the integration limits $\alpha=0$ and $\alpha=1$, and a number of simple expressions for $\Delta \mathrm{A}$ can be derived. We illustrate in the Fig. that the assumption of linearity can be valid with remarkable accuracy, but see $e . g$. ref.[11] for a counterexample. Three expressions that are exact in the linear regime, and that have been used in our previous work are:

$$
\begin{aligned}
\Delta A & =\frac{1}{2}\left(\Delta E_{0}+\Delta E_{1}\right) \\
& =\Delta E_{0}-\frac{\sigma_{0}^{2}}{2 k T} \\
& =\Delta E_{1}+\frac{\sigma_{1}^{2}}{2 k T}
\end{aligned}
$$

where the first expression is a two-point estimate of the integral, and the latter two expressions are obtained from integrating the surface under a straight line through either the initial or the final point, with a slope given by the first derivative of the integrand in that point:

$$
\begin{aligned}
\frac{\mathrm{d}}{\mathrm{d} \alpha}\left[\left\langle\Delta E\left(\left\{R_{i}\right\}\right)\right\rangle_{\alpha}\right] & \\
& =-\frac{1}{k T}\left[\left\langle\Delta E\left(\left\{R_{i}\right\}\right)^{2}\right\rangle_{\alpha}-\left\langle\Delta E\left(\left\{R_{i}\right\}\right)\right\rangle_{\alpha}^{2}\right] \\
& =-\frac{1}{k T} \sigma_{\alpha}^{2}
\end{aligned}
$$

The latter expression shows that the slope of the integrand is proportional to the variance (fluctuations) of the vertical energy. The assumed linear behavior of $\Delta \mathrm{E}_{\alpha}$ implies that the first derivative is constant, and that all higher derivatives vanish. While this leads trivially to the property that $\sigma_{0}^{2}$ equals $\sigma_{1}^{2}$, it is a lengthier derivation, beyond the scope of this paper, to show that this leads to a Gaussian probability distribution of $\Delta \mathrm{E}$. The corresponding free energy profile, given by $-\mathrm{kT}$ times the logarithm of this probability distribution, is parabolic, and the solvent reorganization energy $(\lambda)$ can directly be associated with the fluctuations as

$$
\lambda=\frac{\sigma_{0}^{2}}{2 k T}=\frac{\sigma_{1}^{2}}{2 k T}=\frac{1}{2}\left(\Delta E_{0}-\Delta E_{1}\right)
$$

where the last equality is obtained by subtracting the last two equations for $\Delta \mathrm{A}$.

In the remainder of this paper, we will use the above equations in a simple threestep recipe to compute $\Delta \mathrm{A}$ and $\lambda$ :

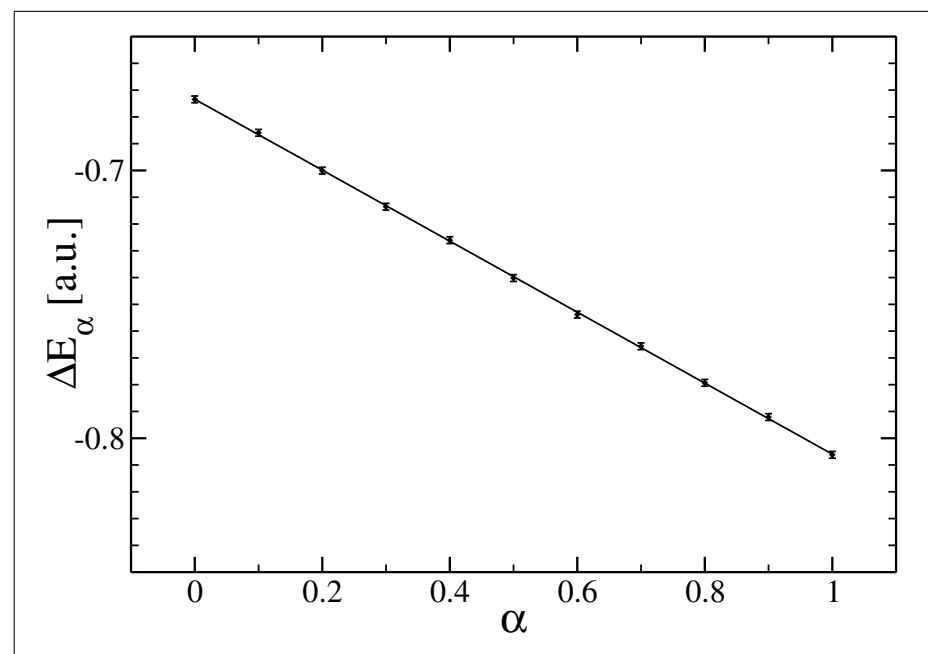

i) Generate an ensemble of atomistic configurations by running molecular dynamics simulations in the reduced $(\alpha=$ $0)$ and/or the oxidized state $(\alpha=1)$.

ii) For each of these configurations, compute the vertical energy $(\Delta \mathrm{E})$ as the difference in total energy between the oxidized and the reduced state.

iii) Compute the average $\left(\Delta \mathrm{E}_{\alpha}\right)$ and variance $\left(\sigma_{\alpha}{ }^{2}\right)$ of the set of values of $\Delta E$ to obtain $\Delta \mathrm{A}$ and $\lambda$.

Additionally, based on careful considerations of the system's complexity, we will choose which formula for $\Delta \mathrm{A}$ and $\lambda$ we employ, and how we generate the ensemble. For example, the expressions depending on the variance of $\Delta \mathrm{E}$ converge significantly slower than those depending only on the average of $\Delta \mathrm{E}$, but have nevertheless the advantage that they can be evaluated with just one simulation in an oxidation state of choice. The expression based on the average of the vertical energy at both end points is likely to be more reliable if some deviation from linearity is to be expected.

Finally, we conclude this section with a brief discussion of our computational setup, referring to ref. ${ }^{[23]}$ for a complete technical review of the method, and refs. ${ }^{[10,12,15]}$ for specific computational details for each of the selected applications. The unifying theme for the simulations that we have selected for this paper is that all DFT calculations have been performed using the freely available simulation package CP2K/Quickstep. ${ }^{[23,24]}$ Based on the hybrid Gaussians and plane waves (GPW) scheme, ${ }^{[25]}$ excellent efficiency and accuracy is obtained for systems containing up to a few thousand atoms.[26,15] The efficiency is obtained by exploiting the locality and compactness of a Gaussian basis, and the linear scaling cost of evaluating the Coulomb (Hartree) energy in a plane wave basis. Furthermore, BornOppenheimer molecular dynamics simulations can be performed using a robust wavefunction optimization technique ${ }^{[26]}$ and a density matrix extrapolation scheme.[23]
Fig. Computed values of the integrand $\Delta \mathrm{E}_{\alpha}$ for a classical model of the $\mathrm{Fe}^{2+} / \mathrm{Fe}^{3+}$ redox pair in aqueous solution are shown with error bars indicating the statistical uncertainty. The line represents a linear fit to the data. The high quality of this fit convincingly demonstrates, for this system, the validity of a key assumption underlying Marcus theory and our computational approach. 
The value of this approach can be best appreciated for 'electronically difficult' systems such as radicals and transition metal compounds, typically encountered in ET systems, where these methods bring enhanced stability.

Nevertheless, these simulations remain challenging and a number of issues that might affect their accuracy have been discussed in more detail in ref. ${ }^{[14]}$ Errors arise from the approximate nature of DFT and from the limited length and timescales that can be assessed by $a b$ initio techniques. The most serious DFT error is likely to come from the self-interaction error, despite the fact that our half-cell approach avoids the difficulties associated with a computational setup where both donor and acceptor are present in the same simulation cell. The latter setup can lead to an unphysical delocalization of the electron and requires proper treatment. However, even with the half cell approach, the selfinteraction error is a major concern for systems containing an unpaired electron in an electronic state that is (nearly) degenerate with the band of occupied solvent state. In this case, an unphysical delocalization of the spin over the solvent might be observed.[27] System size effects can be expected for quantities, such as $\lambda$, that are sensitive to the long-range nature of the electrostatics, since charged solutes are treated in relatively small simulation cells. However, when investigating differences between systems that have a similar spatial distribution of the charge, the same unit cell, and a similar environment, these errors are expected to cancel. Finally, in assuming a linear response regime for our calculations, we have introduced a systematic error. This is error must be balanced to the statistical uncertainty in the results, since only a relatively small number of configurations (100-1000 s) can be computed using methods based on DFT.

\section{Results and Discussion}

In the following, we present three applications that have been used to explore the capabilities and limits of our methodology within the framework of CP2K. These are:

i) the organosulphur compounds tetrathiafulvalenene (TTF) and thianthrene (TH) in acetonitrile (ACN) solution, $[10]$

ii) model quinones, benzoquinone (BQ) and duroquinone (DQ) in two different solvents, ACN and methanol $(\mathrm{MeOH}),{ }^{[12]}$

iii) two natural varieties of the iron-sulfur protein rubredoxin in aqueous solution. ${ }^{[15]}$

Three variants of the same three-step

Table. Computed reaction free energies in $\mathrm{eV}$ for the full reactions discussed in the text. The statistical uncertainty on the computed results is about $60 \mathrm{meV}$ for the first four and about $30 \mathrm{meV}$ for the last reaction.

$\begin{array}{llllll}\text { Redox reaction } & & \text { solvent } & \text { Ref. } & \begin{array}{l}\text { Computed } \\ {[\mathrm{eV}]}\end{array} & \begin{array}{l}\text { Experiment } \\ {[\mathrm{eV}]}\end{array} \\ \mathrm{TH}+\mathrm{TTF}^{+} & ->\mathrm{TH}^{+}+\mathrm{TTF} & \mathrm{ACN} & {[10]} & 0.96 & 0.93 \\ \mathrm{TH}^{+}+\mathrm{TTF}^{2+} & ->\mathrm{TH}^{2+}+\mathrm{TTF}^{+} & \mathrm{ACN} & {[10]} & 1.09 & 1.08 \\ \mathrm{BQ}^{-}+\mathrm{DQ} & ->\mathrm{BQ}+\mathrm{DQ}^{-} & \mathrm{ACN} & {[12]} & 0.42 & 0.35 \\ \mathrm{BQ}^{-}+\mathrm{DQ} & ->\mathrm{BQ}+\mathrm{DQ}^{-} & \mathrm{MeOH} & {[12]} & 0.43 & 0.42 \\ \mathrm{CpFe}(\mathrm{III})+\mathrm{PfFe}(\mathrm{II}) & ->\mathrm{CpFe}(\mathrm{II})+\mathrm{PfFe}(\mathrm{III}) & \text { Water } & {[15]} & 0.04 & 0.06\end{array}$

recipe have been employed. For the organosulphur compounds, we have employed ab initio molecular dynamics simulations to generate the configurations, leading to parameter-free estimates of the reaction free energies shown in the Table, which agree with experiment to within our estimated statistical uncertainty $(60 \mathrm{meV})$. For the other two applications, configurations have been generated using classical molecular dynamics, and DFT has only been employed to compute the vertical energies. These results are thus not truly parameter-free, since a classical force field must be available to describe the geometries. However, this approach allows much longer timescales to be explored, and both systems have been simulated for several nanoseconds, retaining a few hundred to thousands of configurations for DFT-based analysis. The simulations of the quinones exhibit similar agreement with experiment for the reaction free energies (hence validating our mixed classical/quantum approach), but more interestingly allow the effect of hydrogen bonding on the solvent reorganization free energy to be illustrated, and hence the rate of electron transfer. Indeed, we have selected two solvents (ACN and $\mathrm{MeOH}$ ) with very similar dielectric properties. In particular, their Pekar factors, which in a continuum description are proportional to the solvent reorganization energy, differ only by about $5 \%$. However, we find that the solvent reorganization energies of both solutes are larger by approximately $230 \mathrm{meV}$ in the hydrogen bonding solvent, consistent with experiment.[28] This illustrates the limits of a continuum theory approach, which predicts a much smaller difference. Our third application is also based on classical sampling with DFT calculations of the vertical energies, but applies this technique to a significantly larger system, the mesophilic Clostridium pasteurianum $(\mathrm{Cp})$ and the hyperthermophilic Pyrococcus furiosus (Pf) variants of the iron-sulphur protein rubredoxin. We consider it a significant break-through that we are now able to obtain redox potentials differences in agreement with experiment (see the Table) for a system of this size (2800 atoms). Furthermore, we also obtain solvent reorganization energies (0.5-0.7 $\mathrm{eV}$ ) that are in good agreement with the estimates employed in kinetic models of the self-exchange reaction. ${ }^{[29]}$ This is significant, since simulations based on standard force fields yield results that are much larger. This overestimate is consistent with the continuum dielectric expression of the solvent reorganization energy, and underlines the importance of the high frequency dielectric response. The latter term is absent in non-polarizable force fields, but included in our DFT description.

Finally, we note that atomistic and electronic information is available in these simulations as well. For example, the response of the solvent to the ionization of the solute can be analyzed, ${ }^{[10]}$ the contribution of particular residues to the solvent reorganization energy estimated, $[15,30]$ or the correlation between one-electron energies levels and redox potentials obtained. ${ }^{[14]}$ In this paper, we focused on the computation of key parameters in Marcus theory and compared these results with experiment where available. The results presented here are a good indication that the method is quantitative and predictive, and that our approach can thus be applied in cases where experiments might be difficult or more approximate theories inappropriate.

Received: December 18, 2006

[1] R. A. Marcus, N. Sutin, Biochim. Biophys. Acta 1985, 811, 265.

[2] M. Graetzel. Nature 2001, 414, 338.

[3] I. Tavernelli, R. Vuilleumier, M. Sprik, Phys. Rev. Lett. 2002, 88, 213002.

[4] J. Blumberger, M. Sprik, J. Phys. Chem. B 2004, 107, 6529.

[5] J. Blumberger, L. Bernasconi I. Tavernelli, R. Vuilleumier, M. Sprik, J. Am. Chem. Soc. 2004, 126, 3928. 
[6] J. Blumberger, M. Sprik, J. Phys. Chem. B 2005, 109, 6793 .

[7] Y. Tateyama, J. Blumberger, M. Sprik, I. Tavernelli, J. Chem. Phys. 2005, 122, 234505.

[8] J. Blumberger, Y. Tateyama, M. Sprik, Comp. Phys. Comm. 2005, 169, 256.

[9] J. Blumberger, M. Sprik, Theor. Chem. Acc. 2006, 115, 113.

[10] J. VandeVondele, R. Lynden-Bell, E. J. Meijer, M. Sprik, J. Phys. Chem. B 2006, 110,3614

[11] J. Blumberger, I. Tavernelli, M. L. Klein, M. Sprik, J. Chem. Phys. 2006, 124, 064507.

[12] J. VandeVondele, M. Sulpizi, M. Sprik, Angew. Chem. Int. Ed. 2006, 45, 1936.

[13] R. Ayala, M. Sprik, J. Chem. Theory Comp. 2006, 2, 1403.

[14] J. VandeVondele, R. Ayala, M. Sulpizi, M. Sprik, J. Electroanalytical Chem., doi: 1016/J.Jelechem.2007.01.009.

[15] M. Sulpizi, S. Raugei, J. VandeVondele, P. Carloni, M. Sprik. J. Chem. Phys. B, accepted.

[16] R. A. Marcus, J. Chem. Phys. 1956, 24, 966.

[17] R. A. Marcus, Rev. Mod. Phys. 1993, 65, 599.
[18] A. Warshel, J. Phys. Chem. 1982, 86, 2218.

[19] G. King, A. Warshel, J. Chem. Phys. 1990, 93, 8682.

[20] M. Tachiya, J. Phys. Chem. 1993, 97, 5911.

[21] D. W. Small, D. V. Matyushov, G. A. Voth, J. Am. Chem. Soc. 2003, 125, 7470.

[22] M. H. M. Olsson, G. Hong, A. Warshel, J. Am. Chem. Soc. 2003, 125, 5025.

[23] J. VandeVondele, M. Krack, F. Mohamed, M. Parrinello, T. Chassaing, J. Hutter, Comp. Phys. Comm. 2005, 167, 103.

[24] CP2K can be obtained free of charge from http://cp2k.berlios.de/

[25] G. Lippert, J. Hutter, M. Parrinello, Mol. Phys. 1997, 92, 477.

[26] J. VandeVondele, J. Hutter, J. Chem. Phys. 2003, 118, 4365.

[27] J. VandeVondele, M. Sprik, Phys. Chem. Chem. Phys. 2005, 7, 1363.

[28] T. Yago, Y. Kobori, K. Akiyama, S. TeroKubota, Chem. Phys. Lett. 2003, 369, 49.

[29] P. Kennepohl, E. I. Solomon, Inorg. Chem. 2003, 42, 696.

[30] J. Blumberger, M. L. Klein, J. Am. Chem. Soc. 2006, 128, 1854. 